\section{Optical Diode Based on Core-Shell Quantum Dots Demonstrated}

With sizes in the range of just a few nanometers, quantum dots have become an attractive choice for biological labeling and novel optics. These semiconductor nanocrystals emit light in a particular narrow-wavelength band after an outside source, such as an ultraviolet light, excites the electrons in them. By simply changing the size of the semiconductor core, the emission wavelength can be tuned and particles can be created that fluoresce different colors. By utilizing these properties of CdSe/ZnS core-shell quantum dots, D. Alexander and coworkers at the University of NebraskaLincoln have demonstrated a device with the properties of an optical diode.

For CdSe/ZnS core-shell quantum dots, sizes ranging from $3 \mathrm{~nm}$ to $6 \mathrm{~nm}$ can cover emission wavelengths from $490 \mathrm{~nm}$ to $620 \mathrm{~nm}$. As they reported in the July 1 issue of Optics Letters (p. 1957), the researchers used two different sizes of quantum dots, and while the first type of particles (green) emitted at $540 \mathrm{~nm}$, the other type (red) not only emitted at $620 \mathrm{~nm}$ but also absorbed the emission of green particles efficiently. The dried green quantum dots were loaded into one end of a plastic capillary fiber splice, which had an inner diameter of $140 \mu \mathrm{m}$, and red quantum dots were loaded into the opposite end. Each end of the splice was then coupled to a fiber with a core diameter of $99 \mu \mathrm{m}$ and a cladding diameter of $140 \mu \mathrm{m}$. When a 488-nm argon ion laser with a power of $10 \mathrm{~mW}$ was focused into the fiber, the spectrometer output varied with not only the thicknesses of the two quantum dot layers, but also with which layer was illuminated first by the pump laser, demonstrating properties of an optical diode. For example, when the film thicknesses of both red and green particles were the same, the emission of the device was dominated by the red particles with a peak at $640 \mathrm{~nm}$, regardless of which quantum dot film layer was illuminated first. This is because the red dots absorbed all the pump power and also the emission from the green dots, the researchers said. But when the thickness of the green quantum dot film was twice that of the red dot film, the emission spectrum was dominated by the red dots only when the input laser was incident on the red layer first, said the researchers. If the input laser was incident on the green layer first, then the emission spectrum exhibited a strong fluorescence peak of green dots at $565 \mathrm{~nm}$, with a narrow peak at $488 \mathrm{~nm}$ depicting a significant amount of unabsorbed pump light. The researchers attributed the observed asymmetry to the effects of absorption saturation in the red dots, which can absorb both the 488-nm pump light and the fluorescence from the green dots. According to the researchers, this shows that the output of the device can be either the incoming laser wavelength or the fluorescence wavelength of one of the quantum dots. The researchers said that such devices could have useful applications in optical communications systems.

TUSHAR PRASAD

\section{Silver Metamaterial Engineered with Negative Refraction and Low Loss at Telecom Wavelengths}

In all natural materials, the light-speed slow-down factor, due to the real part of the complex index of refraction, is a positive number greater than one, indicating that light travels more slowly in materials than in vacuum. Over the past few years, however, engineered "metamaterials" have begun to emerge that possess a negative index of refraction. In an article in the June 15, 2006, issue of Optics Letters (p. 1800), G. Dolling from Universität Karlsruhe, C.M. Soukoulis from Iowa State University, S. Linden from Institut für Nanotechnologie in Karlsruhe, and their colleagues report the fabrication of a new metamaterial that displays a nega- tive index of refraction and low loss at telecommunication wavelengths.

Physicists have understood for decades that a material with a negative value of the real part of the index of refraction would have many bizarre properties, such as inverted refraction and a reversed Doppler shift. The first metamaterials to be demonstrated were built from split rings of copper metal arranged in periodic arrays, but these and related composite materials have significant losses and until recently only operated at microwave wavelengths. The results reported by Dolling and co-researchers are for a $100 \mu \mathrm{m} \times 100 \mu \mathrm{m}$ device based on two grids of 45-nm-thick silver wire separated by a 30-nm-thick $\mathrm{MgF}_{2}$ spacer, fabricated on a glass substrate that had a 5-nm-layer of indium tin oxide. The silver wires were $\sim 100 \mathrm{~nm}$ wide along one grid direction and $\sim 316 \mathrm{~nm}$ wide along the other grid direction. By measuring the transmission and reflection characteristics of the device between $900 \mathrm{~nm}$ and $1700 \mathrm{~nm}$, the research team was able to extract the real and imaginary parts of the refractive index, corresponding respectively to the effects of refraction and absorption. The absorption is nonnegligible, but the index of refraction is negative across a large fraction of this wavelength range. The ratio of the real

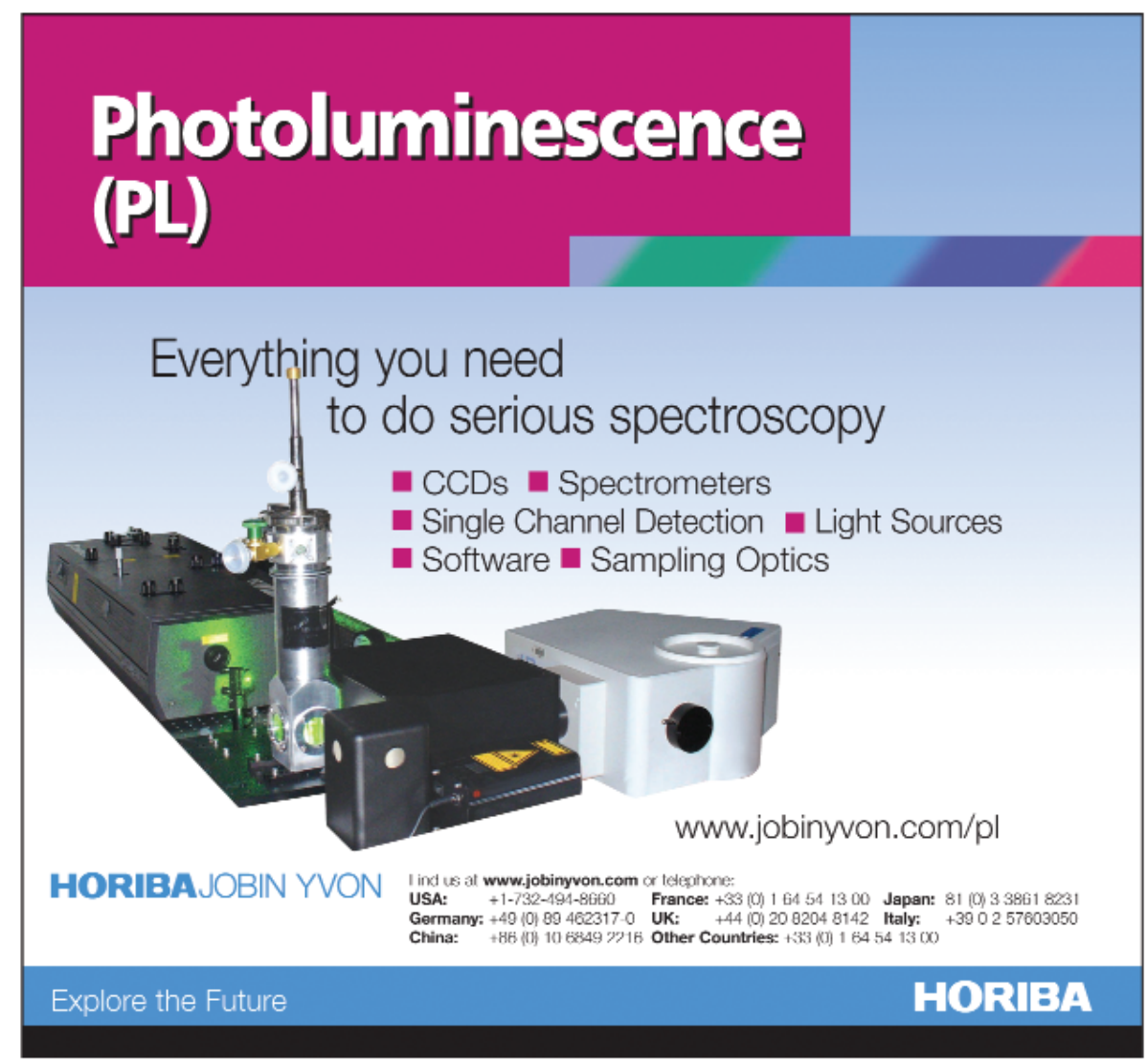

For more information, see http://www.mrs.org/bulletin_ads 